\title{
Successful management of refractory ulcerative colitis with orally administered serum-derived bovine immunoglobulin therapy
}

\author{
Brian D. Beauerle ${ }^{1}$, Bruce P. Burnett ${ }^{2 *}$ and Gerald W. Dryden ${ }^{1}$ \\ ${ }^{1}$ School of Medicine-University of Louisville, Louisville, KY, USA \\ ${ }^{2}$ Entera Health, Inc., Medical Affairs, Cary, NC, USA
}

\begin{abstract}
This case describes a female patient with a long history of pan-colitis ( $>10$ years) with a recent flare of disease precipitated by a Clostridium difficile infection (CDI) after experiencing septic arthritis. The CDI was successfully treated with vancomycin confirmed by a negative PCR test. The patient then experienced a flare of ulcerative colitis (UC) with 7-8 watery, bloody stools per day with cramping. Initial treatment with $30 \mathrm{mg}$ oral prednisone daily and rectal mesalamine, $1 \mathrm{~g}$ QHS, at bedtime resulted in no change in condition. Increased oral steroid (40 mg q.d.) caused an increase of watery, bloody stools to $10-15$ per day. The patient was advised to include oral serum-derived bovine immunoglobulin/protein isolate (SBI), a prescription medical food, in combination with oral steroids. Over two months, this combination effectively resolved gastrointestinal symptoms and a repeat flexible sigmoidoscopy demonstrated mucosal healing (from Mayo UC score grade 2 to grade 0 ). The steroid dose was tapered from $40 \mathrm{mg}$ to $5 \mathrm{mg}$ in combination with SBI and the patient continues to be maintained on both therapeutics for a year. We discuss the unique complications of managing this particular patient, her refusal of anti-TNF therapies and why SBI was a therapeutic option in this case.
\end{abstract}

\begin{abstract}
Abbreviations: ASA: 5-aminosalicyclic acid; CDI: Clostridium difficile infection; DNA: deoxyribonucleic acid; CMV: human cytomegalovirus; GI: gastrointestinal; IBD: inflammatory bowel disease; IV: intravenous; PCR: polymerase chain reaction; QHS: every night at bedtime; q.d.: once daily; q.i.d.: four times a day; SBI: serum-derived bovine immunoglobulin/protein isolate; TNF: tumor necrosis factor; UC: ulcerative colitis; UCDAI: ulcerative colitis disease activity index
\end{abstract}

\section{Introduction}

Ulcerative colitis (UC) is an immune disorder characterized by chronic, colonic inflammation presenting with bloody diarrhea, tenesmus and urgency [1]. The exact pathogenesis of inflammatory bowel disease (IBD), like UC, continues to be delineated, however diet, gut microbiota and genetic susceptibility are jointly implicated [2]. Clostridium difficile is a spore-forming, toxin-producing bacterium which causes a range of symptoms from mild to explosive diarrhea as well as pseudomembranous colitis [3]. It is well-known that patients with UC are more susceptible to colonic infections, in particular C. difficile infection (CDI) [3]. In addition, CDI outcomes for IBD patients are worse and include elevated recurrence, higher rates of colectomy and death. Although it is unknown exactly why there is an association between CDI and IBD, it could be due to a myriad of factors including recurrent hospitalizations for IBD with repeated exposure to $C$. difficile, immunomodulatory drugs which alter the intestinal microbial population to a favorable environment for $C$. difficile as well as altered nutritional status [3].

Refractory UC is challenging for the clinician. Management of UC is particularly difficult when specific therapeutic options are rejected by the patient or deemed unadvisable due to recent co-morbidities, such as CDI. We present a patient with a greater than 10 year history of pan- colitis who had a flare of disease precipitated by CDI. The patient was refractory to escalating oral steroid dose, refused further steroid dose increase and infusions as well as reinitiation of anti-TNF therapy after CDI, secondary to broad-spectrum antibiotic use for the treatment of a septic arthritic knee. The patient was advised to include a prescription medical food, EnteraGam ${ }^{\circledR}$, which contains serum-derived bovine immunoglobulin/protein isolate (SBI) as part of her therapeutic regimen. The nutritional agent was prescribed due to its unique mechanism of action which includes maintaining gastrointestinal (GI) immune balance and managing gut barrier function [4]. After eight weeks of SBI and oral steroids, the patient denied further bleeding, loose stools or abdominal cramping and a repeat flexible sigmoidoscopy illustrated quiescent colitis.

\section{Case report}

A 60-year-old $1.7 \mathrm{~m}, 99.8 \mathrm{~kg}$, Caucasian female was seen in clinic reporting 7 to 8 watery, bloody bowel movements daily and complaining of cramping. The patient had been followed for over 10 years for treatment of pan-colonic UC. Prior to the current illness, the patient had maintained remission of her colitis symptoms for two years with anti-TNF therapy (adalimumab). However, adalimumab was discontinued upon development of septic arthritis in the left knee. To treat the infection, she was placed on long-term antibiotics

Correspondence to: Bruce P. Burnett, $\mathrm{PhD}$, Entera Health, Inc., Medical Affairs, 2000 Regency Parkway, Ste. 255, Cary, NC 27518, USA, Tel: 954-299-1105, E-mail: bruce.burnett@enterahealth.com

Key words: ulcerative colitis, serum bovine immunoglobulin, C. difficile, diarrhea Received: April 18, 2015; Accepted: May 18, 2015; Published: May 20, 2015 
and underwent multiple wash-out surgeries. During the antibiotic courses, the patient developed CDI twice. Oral vancomycin was used to treat both occurrences of CDI; however, only the second course of vancomycin improved the patient's symptoms. She returned threemonths later complaining of the aforementioned 7 to 8 watery, bloody stools and cramping. CDI was not suspected since $C$. difficile testing by PCR was negative. Rather, a UC flare was considered the most likely cause. A flexible sigmoidoscopy was performed, revealing Mayo UC grade 2 colitis (Figure 1A). In addition, there was no evidence of human cytomegalovirus (CMV) in biopsies. Patient comorbidities included: gastroesophageal reflux disease, hypertension, hyperlipidemia, and deep vein thrombosis complicated by a pulmonary embolism. A treatment regimen of oral prednisone, $30 \mathrm{mg}$ daily, and rectal mesalamine, $1 \mathrm{~g}$ at bedtime (QHS), was added to her current regimen of oral mesalamine. Prednisone was increased to $40 \mathrm{mg}$ daily in the absence of clinical improvement. However, her stool frequency increased to 10 to 15 watery stools per day, accompanied by frequent bleeding during the approximately 3 week period on the higher steroid dose. Although a biologic agent was recommended, due to her history of septic arthritis, the patient refused. She also refused high-dose IV corticosteroids because she did not want to be admitted to the hospital. Surgery was recommended if symptoms continued to worsen.

At this point, oral SBI at $5 \mathrm{~g}$ four times daily (q.i.d) was added to the steroid therapy for one week. After the first week, the SBI dose was decreased to $5 \mathrm{~g}$ daily. At the follow-up, four-weeks after initiating SBI, the patient reported 1 to 2 formed bowel movements per day. She denied rectal bleeding or abdominal cramps. Over the next six weeks, prednisone was tapered from 40 to $5 \mathrm{mg}$. An erythrocyte sedimentation rate, initially measured at $90 \mathrm{~mm} / \mathrm{h}$, decreased to $11 \mathrm{~mm} / \mathrm{h}$ over four months. Other bloodwork normalized as well: her white blood cell count decreased from 12.4 to 10.9 thousand/ $\mu \mathrm{L}$; hemoglobin increased from 10.9 to $12.4 \mathrm{~g} / \mathrm{dL}$; hematocrit increased from $34.7 \%$ to $38.0 \%$ and a platelet count decreased from 482 to 248 thousand/ $\mu \mathrm{L}$. Serum albumin concentration increased from $4.1 \mathrm{mg} / \mathrm{dL}$ to $4.5 \mathrm{mg} / \mathrm{dL}$. C. difficile toxin $\mathrm{B}$ remained negative by PCR. A repeat flexible sigmoidoscopy demonstrated normal colonic mucosa approximately two months after beginning SBI therapy (Figure 1B). The patient remains asymptomatic on SBI after one year, maintained only on a dose of $5 \mathrm{~g}$ SBI daily.

\section{Discussion}

This case could appear routine insofar that patients with UC are more susceptible to CDI [3]. However, at the third presentation of 7-8 loose and bloody stools per day, CDI was ruled out due to negative PCR. Active colitis was confirmed with a flexible sigmoidoscopy and

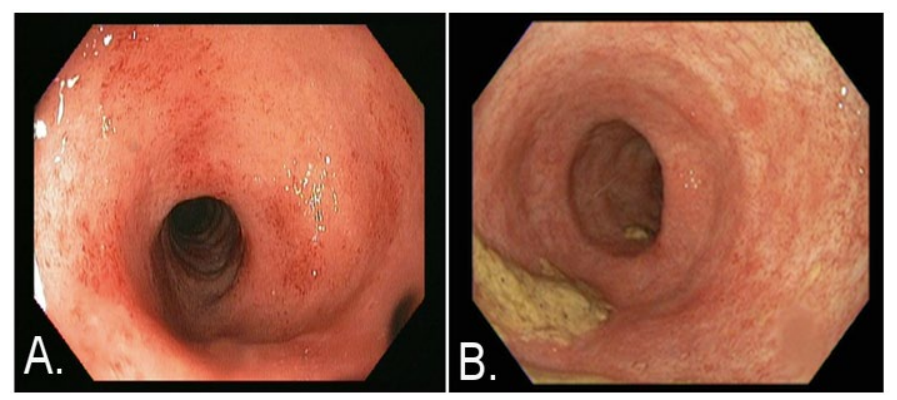

Figure 1. Images obtained via flexible sigmoidoscopy at time of presentation A) and approximately two months after SBI administration B). At the time of presentation patient had Mayo ulcerative colitis (UC) grade 2 score and after two months of ingesting SBI while tapering prednisone, the patient had grade 0 score.

Units of Measurement: All units of measurement are in metric units. biopsies, more than likely due to a precipitation of disease flare by CDI [3]. Yet, as the patient was treated for her UC flare, she was refractory to oral steroids. She also refused to reinitiate any anti-TNF agent, which had previously managed her condition for two years, or high-dose IV corticosteroids since she did not want to be hospitalized. The patient's refusal of therapy was attributed to the septic arthritic knee and to a fear of repeat CDI if she were hospitalized, respectively.

As far as other therapeutic considerations, medicinal agents that affect intestinal bacteria, such as antibiotics and probiotics, have been shown to result in altered symptomology and severity of UC. In various trials, there are examples of successfully using single antibiotics in refractory UC $[5,6]$. Recently, a triple-antibiotic regime has been shown in an open-label, multicenter study to be effective in steroid-free remission of moderate-to-severe UC [7]. Since this patient's UC flare was precipitated by a CDI due to long-term antibiotic use, initiating antibiotics could have led to another CDI. It is also known that the probiotic blend VSL\#3 (Bifidobacterium breve, Bifidobacterium longum, Bifidobacterium infantis, Lactobacillus acidophilus, Lactobacillus plantarum, Lactobacillus paracasei, Lactobacillus bulgaricus and Streptococcus thermophiles) has been shown to reduce UC disease activity index (UCDAI) scores in mild-to-moderate relapsing UC patients who were being treated with 5-aminosalicyclic acid (ASA) and/or immunosuppressants. Instead of probiotics, this patient was advised to add a prescription medical food, oral SBI (EnteraGam $\left.{ }^{\circledR}\right)$ into her therapeutic regimen.

SBI was chosen as add-on therapy for the following reasons: 1 . As a medical food product, SBI has GRAS (generally recognized as safe) status with food-level safety; 2. There are no known drug interactions and the adverse events are infrequent and mild [8]; 3. SBI has been shown in pre-clinical mouse models of DSS-induced colitis to significantly attenuate mucosal damage [9]; and 4.The mechanism of action for SBI is unique.

The mechanism for SBI begins with the immunoglobulin content in SBI, which survives past the stomach, binding to various microbial breakdown components within the GI tract [4]. This has downstream effects, including maintaining GI immune balance and managing gut barrier function. A recent co-culture model suggests that SBI dampens immune activation through steric exclusion whereby the antigen is bound by the immunoglobulin forming a complex which is too large to penetrate into the lamina propria through tight junctions [10]. This model also demonstrated that SBI also potentially works through immune exclusion such that the antigen-bound, immunoglobulin complex prevents dendritic cells from sampling the antigen even if the epithelial damage is present and the complex penetrates into the lamina propria. In addition, a version of SBI has been used in large farming operations for over twenty-years for early weaning of piglets that suffer severe enteropathy, diarrhea and enteric infections [11]. Clinical studies suggest that the proteins in SBI provide for a unique dietary requirement in intestinal disorders [12]. The GRAS distinction of SBI as reviewed by the FDA suggests that this therapy can be safely used for chronic administration. The most commonly reported adverse events in clinical studies (incidence 2-5\%) included mild nausea, constipation, stomach cramps, headache, and increased urination [8]. Although there are not yet formal randomized, controlled clinical trials with SBI in patients with UC, SBI was chosen over probiotics or antibiotics due to these reasons.

Finally, it is known that steroid-refractory UC patients have a high incidence of human cytomegalovirus (CMV) [13-16]. It has also 
been shown that resistance to steroid treatment can be predicted by the CMV DNA load within inflamed intestinal tissues. However, in our case, the patient's biopsies revealed no signs of CMV; therefore she was not diagnosed or treated as CMV. The supposition that CDI induced a UC flare was the most likely explanation for the patient's symptoms and SBI added to the therapeutic regimen helped to manage the patients overall condition.

\section{Conclusion}

In conclusion, we report the case of a patient with refractory UC who incorporated a non-antibiotic, non-probiotic and GRAS-affirmed medical food containing SBI into her therapeutic regimen. After two months, this patient had a vastly improved Mayo UC score, including the resolution of endoscopic evidence of disease, as visualized through flexible sigmoidoscopy. She also experienced a corresponding decrease in inflammatory markers. The clinical symptoms of stool frequency, loose consistency, rectal bleeding and cramping resolved. The patient has been maintained on SBI 5 g q.d. for a year. This case highlights the utility of including a dietary therapeutic, SBI, with standard medical agents for patients with specific intestinal disorders. SBI was most likely efficacious in this patient due to binding of microbial breakdown components, modulating downstream immune activity in the GI tract along with managing gut barrier function [4]. This case suggests that SBI administration should be considered as add-on therapy when dealing with refractory UC patients. Formal, randomized clinical trials are needed to demonstrate the individual efficacy of SBI for induction of clinical remission and endoscopic healing in IBD.

\section{Informed consent}

Written, informed consent was obtained by the patient and is on-file.

\section{Authorship and contributorship}

All authors made significant contributions to the ideation and interpretation of the case as well as the writing of the manuscript. All authors have also approved the final manuscript. GW Dryden is the article guarantor and the senior author. BP Burnett is the corresponding author.

\section{Acknowledgements}

Writing Assistance - Editing of this manuscript was provided by Victoria Jasion, PhD, Raymond Panas, PhD and Hayley Young, PhD. Drs. Panas and Young are employees of Entera Health, Inc., Cary, NC.

Funding Information: No funding was provided for this case report.

\section{Competing interest}

BD Beauerle has no conflict of interest. GW Dryden is a member of the Speakers Bureau and is an Advisor for Entera Health, Inc. BP Burnett is a salaried employee of Entera Health, Inc., which markets serum-derived bovine immunoglobulin/protein isolate (SBI).

\section{References}

1. Kornbluth A, Sachar DB; Practice Parameters Committee of the American College of Gastroenterology (2010) Ulcerative colitis practice guidelines in adults: American College Of Gastroenterology, Practice Parameters Committee. Am J Gastroenterol 105: 501-523. [Crossref]

2. Leone VA, Cham CM, Chang EB (2014) Diet, gut microbes, and genetics in immune function: can we leverage our current knowledge to achieve better outcomes in inflammatory bowel diseases? Curr Opin Immunol 31: 16-23. [Crossref]

3. Nitzan O, Elias M, Chazan B, Raz R, Saliba W (2013) Clostridium difficile and inflammatory bowel disease: role in pathogenesis and implications in treatment. World J Gastroenterol 19: 7577-7585. [Crossref]

4. Petschow BW, Burnett B, Shaw AL, Weaver EM, Klein GL (2014) Serum-derived bovine immunoglobulin/protein isolate: postulated mechanism of action for management of enteropathy. Clin Exp Gastroenterol 7: 181-190. [Crossref]

5. Turunen UM, Färkkilä MA, Hakala K, Seppälä K, Sivonen A, et al. (1998) Long-term treatment of ulcerative colitis with ciprofloxacin: a prospective, double-blind, placebocontrolled study. Gastroenterol 115: 1072-1078. [Crossref]

6. Gionchetti P, Rizzello F, Ferrieri A, Venturi A, Brignola C, et al. (1999) Rifaximin in patients with moderate or severe ulcerative colitis refractory to steroid-treatment: a double-blind, placebo-controlled trial. Dig Dis Sci 44: 1220-1221. [Crossref]

7. Kato K, Ohkusa T, Terao S, Chiba T, Murakami K, et al. (2014) Adjunct antibiotic combination therapy for steroid-refractory or -dependent ulcerative colitis: an openlabel multicentre study. Aliment Pharmacol Ther 39: 949-956. [Crossref]

8. EnteraGam ${ }^{\circledR}$ Prescribing Information (02/15), http://www.enteragam.com/assets/lib/ EnteraGam-full-prescribing-info-3.pdf, accessed 05/20/2015.

9. Henderson AL, Brand MW, Darling RJ, Maas KJ, Detzel CJ, et al. (2015) Attenuation of colitis by serum-derived derived bovine immunoglobulin/protein isolate in a defined microbiota mouse model. Dig Dis Sci 2015 May 31. [Crossref]

10. Detzel CJ, Horgan A, Henderson AL, Petschow BW, Warner CD, et al. (2015) Bovine immunoglobulin/protein isolate binds pro-inflammatory bacterial compounds and prevents immune activation in an intestinal co-culture model. PLoS One 10: e0120278. [Crossref]

11. Torrallardona D (2010) Spray Dried Animal Plasma as an Alternative to Antibiotics in Weanling Pigs - A Review. Asian-Aust J Ani Sci 23:131-148.

12. Petschow BW, Burnett BP, Shaw AL, Weaver EM, Klein GL (2015) Dietary requirement for serum-derived bovine immunoglobulins in the clinical management of patients with enteropathy. Dig Dis Sci 60: 13-23. [Crossref]

13. Roblin X, Pillet S, Oussalah A, Berthelot P, Del Tedesco E, et al. (2011) Cytomegalovirus load in inflamed intestinal tissue is predictive of resistance to immunosuppressive therapy in ulcerative colitis. Am J Gastroenterol 106: 2001-2008.[Crossref]

14. Pillet S, Pozzetto B, Jarlot C, Paul S, Roblin X (2012) Management of cytomegalovirus infection in inflammatory bowel diseases. Dig Liver Dis 44: 541-548. [Crossref]

15. Ciccocioppo R, Racca F, Paolucci S, Campanini G, Pozzi L, et al. (2015) Human cytomegalovirus and Epstein-Barr virus infection in inflammatory bowel disease: Need for mucosal viral load measurement. World J Gastroenterol 21: 1915-1926. [Crossref]

16. doCarmo AM, Santos FM, Ortiz-Agostinho CL, Nishitokukado I, Frota CS, et al (2014) Cytomegalovirus infection in inflammatory bowel disease is not associated with worsening of intestinal inflammatory activity. PloS One 9:e111574. [Crossref]

Copyright: (C2015 Beauerle BD. This is an open-access article distributed under the terms of the Creative Commons Attribution License, which permits unrestricted use, distribution, and reproduction in any medium, provided the original author and source are credited. 